\title{
A simple analytical model for pressure on obstacles induced by snow avalanches
}

\author{
Thierry FAUG, Benoit CHANUT, Rémi BEGUIN, ${ }^{*}$ Mohamed NAAIM, \\ Emmanuel THIBERT, Djebar BAROUDI \\ Cemagref Grenoble, ETGR, 2 rue de la Papeterie, BP 76, 38402 Saint-Martin-d'Hères Cedex, France \\ E-mail: thierry.faug@cemagref.fr
}

\begin{abstract}
The forces snow avalanches are able to exert on protection dams or buildings are of crucial interest in order to improve avalanche mitigation measures and to quantify the mechanical vulnerability of structures likely to be damaged by snow avalanches. This paper presents an analytical model that is able to calculate these forces taking into account dead-zone mechanisms. First, we present a 2-D analytical hydrodynamic model describing the forces on a wall overflown by gravity-driven flows down an inclined plane. Second, the 2-D model is successfully validated on discrete simulations of granular flows. Third, we provide ingredients to extend the 2-D model to flows of dry and cold snow. Fourth, we propose a simplified 3-D analytical model taking into account lateral fluxes. Finally, the predictions from the simplified 3-D analytical model are successfully compared to recent measurements on two full-scale snow avalanches released at the Lautaret site in France.
\end{abstract}

\section{INTRODUCTION}

The influence of obstacles on avalanche flows has been the topic of many recent studies combining full-scale observations on snow avalanches, small-scale experiments with granular materials, theory and numerical modelling. Studies refer to avalanche flows interacting with deflecting dams (Irgens and others, 1998; Jóhannesson, 2001; Hákonardóttir and Hogg, 2005; Cui and others, 2007; Faug and others, 2007; Gray and Cui, 2007), catching dams (Chu and others, 1995; McClung and Mears, 1995; Naaim and others, 2004; Gauer and others, 2007, 2009; Faug and others, 2008a,b) and retarding mounds (Hákonardóttir and others, 2003; Chiou and others, 2005). Up to now the particular situation of free-surface flows overflowing a catching dam has been addressed in terms of the run-out shortening downstream of the dam (Faug and others, 2008a,b). This situation is of crucial interest with regard to the residual risk downstream of protection dams. Here we consider the force these avalanche flows are able to exert on a flat obstacle when overflowing it, which is also important when estimating the mechanical vulnerability of buildings and protection dams. Recent full-scale observations on snow avalanches report large pressures at low incoming Froude numbers on tubular pylons (Sovilla and others, 2008b,a) and on a flat obstacle (a $1 \mathrm{~m}^{2}$ plate) (Sovilla and others, 2008b; Thibert and others, 2008). Complex materials, such as granular materials and dry snow, can behave as a fluid or a solid: their ability to undergo a transition from a fluid state to a solid state can lead to the formation of stagnant zones when the flow encounters a topographic discontinuity of the ground. While a part of the incoming material is jammed (solid-like phase) another part of the material remains in a fluid-like phase and is deflected, being able to overflow the obstacle. The ability of a granular fluid to form stagnant zones, called granular 'dead zones' (Faug and others, 2002, 2008a; Gray and others,

\footnotetext{
*Present addresses: Laboratoire d'Etude des Transferts en Hydrologie et Environnement, BP 53, 38041 Grenoble Cedex 09, France. Cemagref Aix-en-Provence-Equipe Hydrologie Division OHAX, 3275 route Cézanne, CS 40061, 13182 Aix-en-Provence Cedex 05, France.
}

2003), upstream of a topographic discontinuity may lead to severe modifications of the resulting force on the obstacle. The size of the stagnant zone formed upstream of a catching dam is very large if the catching dam prevents side flows. In this paper, we present a simple analytical model based on momentum balance over a control volume upstream of a flat obstacle in order to analyse and quantify the effect of the dead-zone mechanism on the resulting force. The analytical model is first developed for two-dimensional (2-D) flows and validated in a discrete numerical model for dry granular flows overflowing a dam. Asymptotic behaviours at low and high Froude numbers are discussed. We then extrapolate the model to 2-D dry-snow flows using a Voellmy friction law and adapt the model to a more complex three-dimensional (3-D) geometry, taking into account lateral fluxes. Finally we compare the predictions of the 3-D simplified analytical model to field data available from a $1 \mathrm{~m}^{2}$ plate subject to the impact of snow avalanches, at the Lautaret test site in France. It is shown that our analytical model is able to correctly reproduce the measured force values within the experimental uncertainty found in related previous studies (Thibert and others, 2008; Baroudi and Thibert, in press).

\section{MODELLING OF FREE-SURFACE FLOWS OVERFLOWING A DAM}

\subsection{General framework equations in 2-D geometry}

We consider a steady incoming free-surface flow of thickness $h$, mean velocity $u$ and mean density $\rho$ down a flat slope with inclination $\theta$, as shown in Figure 1 . A nearly triangular stagnant zone is formed upstream of the dam, as shown in Figure 1. Momentum conservation over a control volume, $V_{0}$, upstream of the dam (pink area in Fig. 1) allows us to show that, in a steady-flow regime, the resulting force, $F$, normal to the dam is the sum of four contributions:

$$
F=F_{u}+F_{\mathrm{h}}+F_{\mathrm{w}}+F_{\mathrm{f}} .
$$

$V_{0}$ is a volume per unit width and we consider forces per unit width. $F_{u}$ is a purely dynamic force resulting 


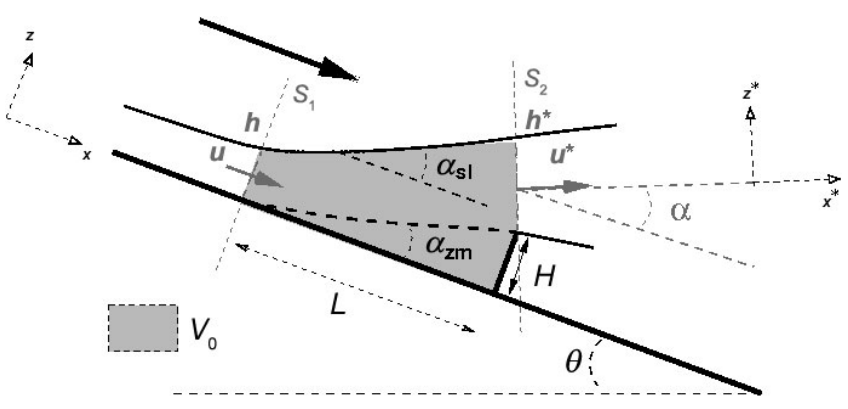

Fig. 1. Sketch of the flow and control volume, $V_{0}$ (pink area). $H$ is the obstacle height; $\alpha_{\mathrm{zm}}$ is the mean angle of the dead zone; $\alpha_{\mathrm{sl}}$ is the mean angle of the free surface above the dead zone; $\alpha$ is the angle between the velocity, $\vec{u}^{*}$, and the ground; $h$ and $u$ are the thickness and the depth-averaged velocity outside the influence area of the obstacle; $h^{*}$ and $u^{*}$ are the thickness and the depthaveraged velocity of the overflow at the top of the dam (in the $x^{*}$ direction); and $L$ is the length of the influence zone upstream of the obstacle, assumed to be close to the length of the dead zone.

from the momentum variation between sections $S_{1}$ and $S_{2}$, defined in Figure 1: $F_{u}=\beta\left(1-\delta_{u} \cos \alpha\right) \rho u^{2} h$, where $\delta_{u}$ is the velocity ratio, $u^{*} / u, u^{*}$ is the velocity at the top of the dam, as defined in Figure 1, and $\alpha$ is the deflection angle (i.e. the angle between $\vec{u}^{*}$ and the ground). The coefficient $\beta$ depends on the shape of the velocity profile and is defined by $\frac{1}{h} \int_{0}^{h} u^{2} \mathrm{~d} z=\beta\left(\frac{1}{\hbar} \int_{0}^{h} u \mathrm{~d} z\right)^{2}$. The relative velocity reduction, $\left(u-u^{*}\right) / u$, is simply assumed to be proportional to the deflection angle, $\alpha$, which gives:

$$
\delta_{u}=1-\kappa \alpha,
$$

where $\kappa$ is a velocity-reduction coefficient. $F_{\mathrm{h}}$ is a purely hydrostatic contribution due to the incoming flow undisturbed by the obstacle, $F_{\mathrm{h}}=\frac{1}{2} k \rho g h^{2} \cos \theta$, where $k$ is the earth pressure coefficient classically introduced for gravitydriven flows of granular materials (Savage and Hutter, 1989) or snow (McClung and Mears, 1995; Bartelt and others, 1999). $F_{\mathrm{W}}$ is the $x$-axis component of the weight of the control volume, $V_{0}: F_{\mathrm{w}}=\rho g V_{0} \sin \theta$. $F_{\mathrm{f}}$ is the basal friction force assumed to be proportional to the $y$-axis component of the weight of the control volume, $V_{0}$ (Coulomb friction): $F_{\mathrm{f}}=\mu_{\mathrm{zm}} \rho g V_{0} \cos \theta$, where $\mu_{\mathrm{zm}}$ is the friction coefficient between the dead zone and the ground. We discuss $\mu_{\mathrm{zm}}$ further below.

The volume, $V_{0}$, can be calculated from Figure 1 as follows:

$$
V_{0} \approx \frac{1}{2}\left\{\left[H+h\left(1+\frac{\delta_{\mathrm{h}}}{\cos \alpha}\right)\right] L-h^{2} \delta_{\mathrm{h}}^{2} \tan \alpha\right\},
$$

where $h^{*}$ is the thickness of the outcoming flow defined in Figure $1, \delta_{\mathrm{h}}$ is the depth ratio, $h^{*} / h, H$ is the obstacle height and $L$ is the length of the influence zone upstream of the obstacle. The zone of influence upstream from the dam is defined as the length of the disturbed flow upstream of the dam (flow depth and velocity are not equal to the incoming flow depth, $h$, and velocity, $u$ ) and can be approximated by $\tan \left(\alpha_{\mathrm{zm}}\right)=H / L$. By mass flow rate conservation, the flow depth ratio, $\delta_{\mathrm{h}}$, is equal to $1 / \delta_{u}$, if we assume that the density is unchanged. The angle $\alpha$ is equal to $\left(\alpha_{\mathrm{sl}}+\alpha_{\mathrm{zm}}\right) / 2$ where $\alpha_{\mathrm{sl}}$ is the angle of the free surface inside the control volume, $V_{0}$. We detail below how we can estimate the angles $\alpha_{\mathrm{zm}}$ and $\alpha_{\mathrm{s}}$.
If we neglect the second-order term $\left(\delta_{\mathrm{h}}^{2} \tan \alpha\right)$ in Equation (3), then Equation (1) can be synthesized in terms of the normalized force, $F /\left(0.5 \rho u^{2} h\right)$, versus the incoming Froude number, $\mathrm{Fr}=u / \sqrt{g h \cos \theta}$ :

$$
\begin{aligned}
& \frac{F}{\frac{1}{2} \rho u^{2} h}=2 \beta[1-(1-\kappa \alpha) \cos \alpha] \\
&+\frac{1}{\mathrm{Fr}^{2}}\left\{k+\left(\frac{\sin \theta-\mu_{\mathrm{zm}} \cos \theta}{\tan \alpha_{\mathrm{zm}}}\right)\right. \\
&\left.\cdot\left[\frac{H}{h}+1+\frac{1}{(1-\kappa \alpha) \cos \alpha}\right] \frac{H}{h}\right\} .
\end{aligned}
$$

The second-order term $\left(\delta_{\mathrm{h}}^{2} \tan \alpha\right)$ is strictly negligible when $h^{*} \ll L$ and $h^{*} \approx h$, which is almost true when the Froude number is not too high (typically less than 5-10). When the Froude number is more than 5-10, the contribution due to the volume, $V_{0}$, in the total force is so weak (purely dynamic force) that an error in $V_{0}$ has no effect on the total resulting force. This analytical model has been initially developed for granular flows (Faug and others, 2009) for which the parameters $\left(k, \beta, \mu_{\mathrm{zm}}, \kappa\right)$ have been determined, as well as expressions to calculate the angles $\alpha_{\mathrm{zm}}$ and $\alpha_{\mathrm{sl}}$, which give the deflection angle, $\alpha$. Results for granular flows are briefly reported in the next subsection.

\subsection{Validation of the analytical model for 2-D granular flows}

We performed discrete-particle simulations using a linear damped spring law between spherical particles with a Coulomb failure criterion, in order to simulate 2-D steady granular flows down an inclined slope, as shown in Figure 1 (see details in Faug and others, 2009). These discrete numerical simulations were based on the moleculardynamics method introduced by Cundall and Strack (1979) and widely used to simulate dense granular flows (Ertas and others, 2001; Silbert and others, 2001; da Cruz and others, 2005). The following microscopic parameters were needed to describe the contacts between grains: the normal and tangential stiffnesses, $k_{\mathrm{n}}$ and $k_{\mathrm{t}}$; the restitution coefficient, $e$ (linked to the damping coefficient) and the interparticle friction coefficient, $\mu$. The influence of these parameters was discussed by Silbert and others (2001): (1) $k_{\mathrm{n}}$ and $k_{\mathrm{t}}$ have no effect in the limit of rigid grains (overlap between particles $<1 / 1000 d$, where $d$ is the particle diameter); (2) $e$ has little effect except for extreme values $e=0$ and $e=1$; (3) $\mu$ has a greater effect on the results, but its influence becomes weak at low values, typically $<0.5$. In our simulations, we used the following values: $k_{\mathrm{n}}=10^{4} \mathrm{~N} \mathrm{~m}^{-1}$, $k_{\mathrm{t}}=1 / 2 k_{\mathrm{n}}, e=0.5$ and $\mu=0.5$ (corresponding to a typical value of the internal friction angle of granular materials). Here we used the commercial code PFC2D (Itasca Consulting: http://www.itasca.com/pfc/index.php). These numerical simulations allowed us to estimate the macroscopic empirical laws to close Equation (4) for the case of granular materials. A first result of the numerical simulations is that the basal friction, $\mu_{\mathrm{zm}}$, was shown to be constant, equal to $\tan \theta_{\min }$, where $\theta_{\min }$ is the minimal angle below which no steady flow is possible (stopping of the flow) (Pouliquen, 1999; GDR MiDi, 2004). Second, the mean angle of the dead zone with the horizontal, $\theta-\alpha_{\mathrm{zm}}$, was shown to be equal to $\theta_{\min }$ for all slope inclinations, $\theta$. The length, $L$, of the influence zone of the obstacle was then assumed to be equal to the length of the stagnant zone and accordingly was defined as: 
$\tan \left(\theta-\theta_{\min }\right)=H / L$. Third, the free-surface angle, $\alpha_{\mathrm{sl}}$, was shown to be a simple linear function of the slope inclination, $\theta: \alpha_{\mathrm{sl}}=a \theta+b$, where $a$ and $b$ are coefficients depending on the incoming flow regime. We defined $\theta_{\max }$ as the maximum angle above which uniform flows were not possible. For uniform flows $\left(\theta_{\min }<\theta<\theta_{\max }\right)$, the angle $\alpha_{\mathrm{sl}}$ was expressed as:

$$
\alpha_{\mathrm{sl}}=\frac{\theta_{\min }}{\theta_{\max }-\theta_{\min }}\left(\theta-\theta_{\min }\right)
$$

For non-uniform flows $\left(\theta>\theta_{\max }\right)$, the angle $\alpha_{\mathrm{sl}}$ was expressed as:

$$
\alpha_{\mathrm{sl}}=\frac{\pi}{2}-\left(\frac{\theta_{\min }-\pi / 2}{\theta_{\max }-\pi / 2}\right)(\pi / 2-\theta) .
$$

Equation (4) was successfully tested on data from discrete numerical simulations using the following values (Faug and others, 2009): $k=1, \beta=5 / 4$ (for a Bagnold-like velocity profile (GDR MiDi, 2004)), $\kappa=(1-e) /(\pi / 2)$ (where $e=0.5$ is the restitution coefficient), $\theta_{\min }=14^{\circ}$ and $\theta_{\max }=24^{\circ}$ (typical values for 2-D granular flows (GDR MiDi, 2004)). The value of $\kappa$ is simply estimated from a purely collisional regime assuming that $u^{*} / u$ scales as $e$ when $\alpha=\pi / 2$ which gives $e=1-\kappa(\pi / 2)$ according to Equation (2). Figure 2 shows predictions of the analytical model compared to discrete simulations. The predictions of the model are in very good agreement with the discrete simulations, without having introduced any fitting parameter. Note that Equation (4) predicts a force ratio $F /\left(0.5 \rho u^{2} h\right)=$ $2 \beta[1-(1-\kappa \alpha) \cos \alpha]$ at high Froude numbers, which gives a value of 2 for $\alpha=\pi / 2$, compatible with the drag coefficient classically given for a flat obstacle in the inertial regime (high Froude number). Figure 2 also reports the force normalized by the purely hydrostatic force, $\frac{1}{2} \rho g h^{2} \cos \theta$. It is interesting to consider the asymptotic prediction for this ratio when $\theta$ tends towards $\theta_{\text {min }}$ (i.e. the Froude number, Fr, tends to zero). According to the analytical model, this ratio should scale as $k+(H / h)(2+H / h)\left(1 / \cos \theta_{\min }\right)$. With $k=1$ and $H / h=1$, this gives a value of $1+3 / \cos \theta_{\min }$. The ratio is then close to $4\left(\cos \theta_{\min } \approx 1\right)$, as found in discrete simulations and shown in Figure 2. In section 2.3, we use the analytical model for dry snow and we provide the model parameters adapted to dry snow.

\subsection{Outlook for 2-D snow flows}

Here we use Equation (4) for dry snow flows and we describe the parameters characterizing the behaviour of dry snow. Cemagref has designed a $10 \mathrm{~m}$ long and $20 \mathrm{~cm}$ wide channel at Col du Lac Blanc, Alpe d'Huez, France, described in detail by Bouchet and others (2003, 2004). Recent investigations on flows of dense and dry snow (for $T<0^{\circ} \mathrm{C}$ ) down this $10 \mathrm{~m}$ long flume showed that snow exhibits some properties similar to those of granular flows (Rognon, 2006; Rognon and others, 2008). Dry and dense snow is a polydisperse granular material. Similarly to granular flows, there exists a minimum angle below which the flow is stopped, and a maximum angle above which flows accelerate along the channel. Steady and uniform flows are only possible between these two angles. Typical values were derived from experimental investigations at Col du Lac Blanc for dense and dry snow (Rognon and others, 2008): $\theta_{\min }=33^{\circ}$ and $\theta_{\max }=42^{\circ}$. These angles were obtained in a narrow channel with typical flow depths $\sim 10 \mathrm{~cm}$. Even if these angles are likely to be influenced by wall effects (whose extent is still under discussion), we will use these angles

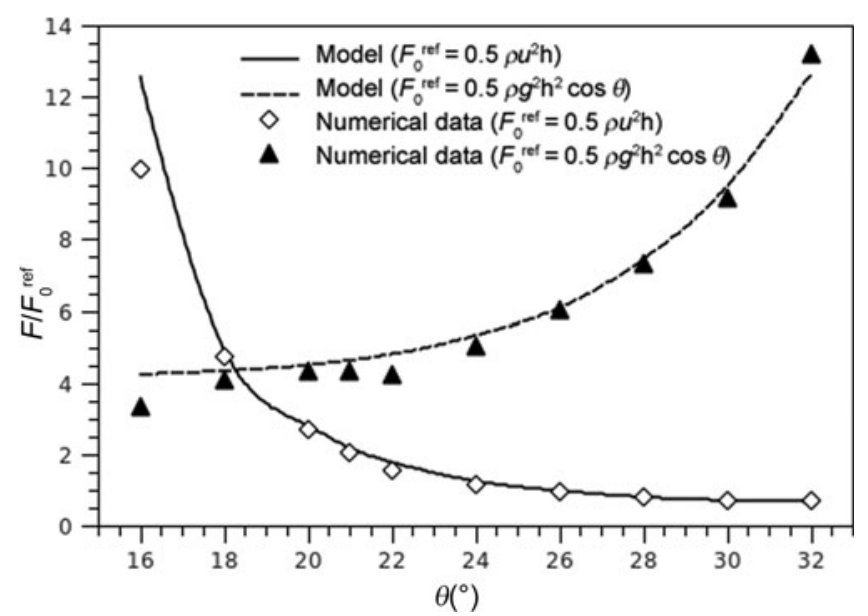

Fig. 2. Granular flows: prediction of the model for the rescaled force, $F /\left(0.5 \rho u^{2} h\right)$ and $F /\left(0.5 \rho g h^{2} \cos \theta\right)$, vs slope, compared to data from numerical discrete simulations. The following parameters were used: $\beta=5 / 4, k=1, \kappa=0.31(e=0.5), \theta_{\min }=14^{\circ}$, $\theta_{\max }=24^{\circ}$ (Faug and others, 2009).

for snow in the following. The flow is divided into two layers: a highly sheared layer of snow grains (typically $1 \mathrm{~mm}$ in size) at the base, surmounted by a low shear layer of aggregates (with a maximum size close to the flow depth). This flow configuration results in a typical velocity profile close to velocity profiles obtained from discrete numerical simulations on bi-disperse granular flows. A value of $\beta$ close to 1 is then reasonable for snow flows.

The friction law for snow flows is still an open question. However, an effective friction law corresponding to a Voellmy model could be fitted to data from the Lac Blanc chute (Rognon, 2006): $\mu=\mu_{\mathrm{s}}+(g / \xi) \mathrm{Fr}^{2}$. The Voellmy model is classically used in snow-avalanche engineering applications (Bartelt and others, 1999); $\mu_{\mathrm{s}}$ is a dry friction coefficient and $\xi$ is a turbulent coefficient. Both parameters $\left(\mu_{\mathrm{s}}, \xi\right)$ were fitted corresponding to various choices. The best fit was obtained for $\mu=0.61$, i.e. $\theta_{\mathrm{s}}=\arctan \left(\mu_{\mathrm{s}}\right)=31.2^{\circ}$, and $\xi=1050 \mathrm{~m} \mathrm{~s}^{-2}$, which are values encountered for snow avalanches (Salm and others, 1990). With respect to the limit angle, $\theta_{\min }=33^{\circ}$, which would imply $\mu=\tan \theta_{\min }$, a value of $\xi=1400 \mathrm{~m} \mathrm{~s}^{-2}$ was obtained.

We assume that the empirical laws derived from investigations on granular flows to estimate the free-surface angle, $\alpha_{\mathrm{sl}}$, the dead-zone angle, $\alpha_{\mathrm{zm}}$, and consequently the deflection angle, $\alpha$, as well as the friction, $\mu_{\mathrm{zm}}$, still hold for our granular-like snow flows. A value of $k=1$ is chosen. Here, $\kappa$ is calculated using a very low value for $e$ that is more compatible with the properties of snow material: $e=0.1$ gives $\kappa=0.57$.

The angle $\alpha$ is assumed to be equal to:

$$
\alpha=\frac{\alpha_{\mathrm{zm}}+\alpha_{\mathrm{sl}}}{2}
$$

where $\alpha_{\mathrm{sl}}$ is defined by Equations (5) and (6).

In steady and uniform flow conditions, or within a flow regime for which the effect of acceleration terms (time-derivative terms in momentum conservation) can be neglected, we have the following equation corresponding to equilibrium between gravity and friction forces $(\tan \theta=\mu)$ :

$$
\theta=\arctan \left(\tan \theta_{\min }+\frac{g}{\xi} \mathrm{Fr}^{2}\right)
$$



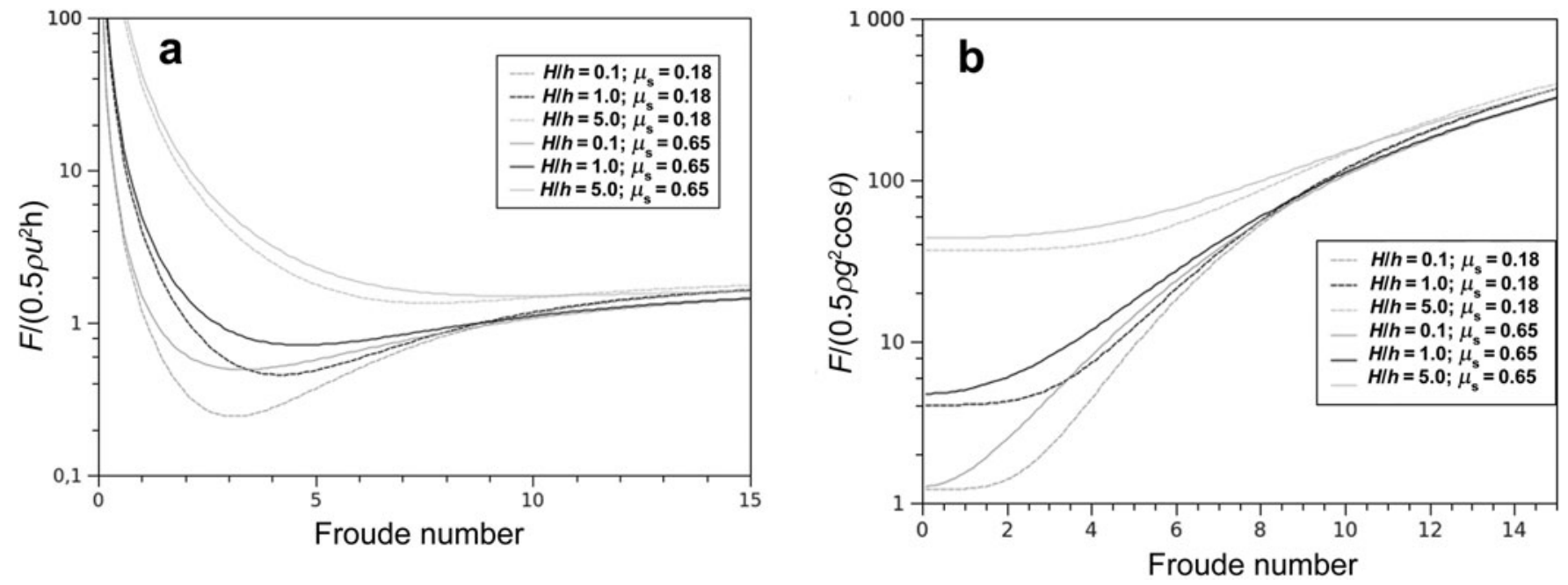

Fig. 3. Snow flows. Prediction of the analytical model for the rescaled forces (a) $F /\left(0.5 \rho u^{2} h\right)$ and (b) $F /\left(0.5 \rho g h^{2} \cos \theta\right)$ vs the Froude number at different ratios, $H / h\left(0.1,1\right.$ and 5). The following parameters were used: $\beta=1, k=1, \kappa=0.57(e=0.1)$ and $\xi=1000 \mathrm{~m} \mathrm{~s}^{-2}$. Predictions are given for two pairs $\left(\theta_{\min }, \theta_{\max }\right)$ keeping $\theta_{\max }-\theta_{\min }=9^{\circ}:\left[33^{\circ} ; 42^{\circ}\right]\left(\mu_{\mathrm{S}}=0.65\right)$ and $\left[10^{\circ} ; 19^{\circ}\right]\left(\mu_{\mathrm{S}}=0.18\right)$.

Equation (8) allows us to eliminate the slope, $\theta$, in Equations (7) and (4) and to express the rescaled force, $F /\left(0.5 \rho u^{2} h\right)$, as a function of the incoming Froude number, $\mathrm{Fr}$, without the prior knowledge of the slope (information which is included in the Froude number). This raises the question whether we may extrapolate the predictions of our analytical model to slope inclinations less than $\theta_{\min }$ in transient conditions towards stopping. The answer to this question is positive if the system is able to reach an equilibrium state for which the assumption $\tan \theta=\mu$ still holds. This assumption seems reasonable in the case of decelerating flows evolving towards stopping (tails of avalanches), for which the effect of time-derivative terms is expected to be negligible. We use this assumption in the following when applying equations established for the steady regime to full-scale avalanche flows.

It is important to note that the rescaled force, $F /\left(0.5 \rho u^{2} h\right)$, depends on the ratio $H / h$. Indeed, attempts to find a relation between this ratio and $\mathrm{Fr}$ are meaningful only if $H / h$ remains constant. Figure 3 gives the prediction of the analytical model in terms of the rescaled force vs Fr for different values of $H / h$, $\theta_{\min }$ and $\theta_{\max }$, keeping $\theta_{\max }-\theta_{\min }$ constant. The curves show that the results are not very sensitive to the value of $\theta_{\mathrm{min}}$. The results are, rather, influenced by the geometry corresponding to a varying ratio, $H / h$. We also show the rescaled force, $F /\left(0.5 \rho g h^{2} \cos \theta\right)$, for the same set of parameters to give the prediction of the analytical model at very low $\mathrm{Fr}$ in order to highlight the transition towards the hydrostatic regime.

\subsection{Outlook for 3-D effects with lateral fluxes}

\subsubsection{General framework equations in 3-D geometry} We propose a modification of Equation (4), taking into account 3-D effects corresponding to lateral fluxes and to the modification of the shape of the dead zone by these lateral fluxes. The resulting analytical model is a simplified model that does not take into account the entire effect of flow spreading, which would require fully 3-D numerical models (e.g. Naaim and others, 2004). Here, we consider the thickness, $h^{\mathrm{L}}$, and the depth-averaged velocity, $u^{\mathrm{L}}$, that correspond to the lateral fluxes around the obstacle. Figure 4 a gives a top view of the flow configuration in 3-D geometry. A dead zone is formed upstream of the obstacle. The base of the dead zone in the plane $(x, y)$ is triangular and is characterized by angle $\gamma$.

Compared to the 2-D geometry, the angles $\alpha_{\mathrm{zm}}, \alpha_{\mathrm{s} \mid}$ and $\alpha$ depend on the transverse position, $y$, along the width, $\ell$, of the obstacle as depicted in Figure $4 \mathrm{~b}$. As a first approximation, and in the absence of well-documented experimental evidence of the shape of the dead zone, we assume a simple triangular shape for the dead zone in a plane normal to the $z$ axis (Fig. 4a), and the length, $L(y)$, at location $y$ can be expressed as $L(y)=c+d y$. The boundary conditions require $0=c+d(\ell / 2)$ (edge of the obstacle) and $H / \tan \left(\theta-\theta_{\min }\right)=c$ (centre of the obstacle), which leads to:

$$
L(y)=\frac{H}{\tan \left(\theta-\theta_{\min }\right)}\left(1-\frac{2}{\ell} y\right) .
$$

The dead-zone angle at position $y$ is $\alpha_{\mathrm{zm}}(y)=$ $\arctan (H / L(y))$. The free-surface angle is assumed to vary linearly between $\pi / 2$ (edge of the obstacle) and the value of $\alpha_{\mathrm{s} \mid}$ in 2-D given by Equations (5) and (6), which gives $\alpha_{\mathrm{sl}}(y)=2\left(\pi / 2-\alpha_{\mathrm{sl}}\right)(y / \ell)+\alpha_{\mathrm{sl}}$. The mean angle of deflection is then given by $\alpha(y)=\left(\alpha_{\mathrm{zm}}(y)+\alpha_{\mathrm{sl}}(y)\right) / 2$. The dependence of these angles on $y$ complicates the calculation of the momentum balance (3-D integrals).

We assume that the basal friction is proportional to the weight in the $z$-axis direction. The total basal force is caused by the basal friction below the dead zone and the sum of the weights of the dead zone and the fluid above the dead zone: $\bar{F}_{\mathrm{w}}-\bar{F}_{\mathrm{f}}=\left(\sin \theta-\mu_{\mathrm{zm}} \cos \theta\right) \rho g \bar{V}$. Here $\bar{V}$ is the control volume in 3-D geometry (between sections $S, S^{*}$ and $S^{\mathrm{L}}$ in Fig. 4):

$$
\begin{gathered}
\bar{V}=\frac{2}{\ell} \int_{0}^{\ell / 2}\left(\frac{1}{2} L(y)\left\{H+h\left[1+\frac{\delta_{\mathrm{h}}(y)}{\cos (\alpha(y))}\right]\right\}\right. \\
\left.-\frac{1}{2} h^{2} \delta_{\mathrm{h}}(y) \tan (\alpha(y))\right) \mathrm{d} y .
\end{gathered}
$$

Equation (10) can be solved numerically and is work in progress. In section 2.4.2 we propose some rough approximations to simplify the model in order to be able to provide an analytical solution under restrictive conditions. 


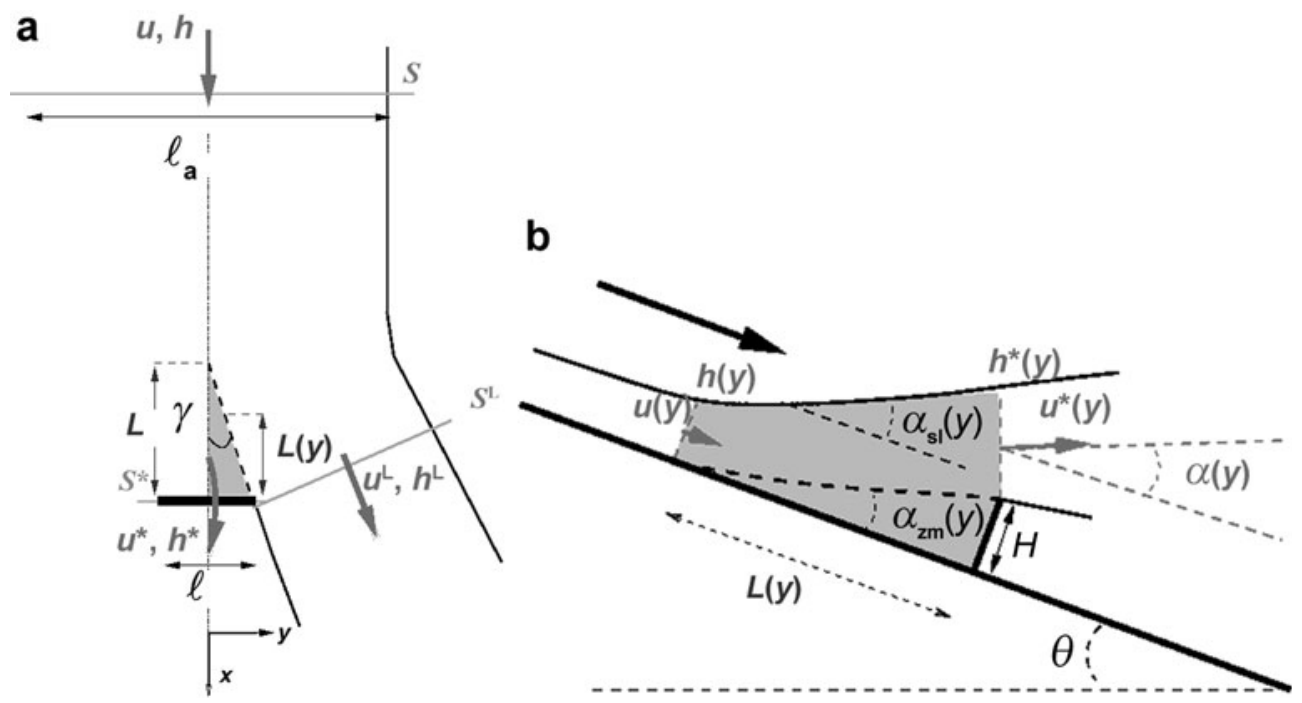

Fig. 4. (a) Top view of the flow and dead zone (pink area) in 3-D geometry. $\gamma$ is the mean angle of the dead zone in the plane $(x, y)$. $h$ and $u$ are the thickness and the depth-averaged velocity outside the zone of influence of the obstacle. $\ell_{\mathrm{a}}$ is the width of the incoming flow. $h^{*}$ and $u^{*}$ are the thickness and the depth-averaged velocity at the top of the dam. $\ell$ is the width of the obstacle. $h^{\mathrm{L}}$ and $u^{\mathrm{L}}$ are the mean thickness and the depth-averaged velocity in the flow branch corresponding to lateral fluxes. $L$ is the length of the dead zone at the centre (in $y$-axis direction) of the obstacle, and $L(y)$ is the length of the dead zone at a given position, $y$. Note that $h^{*}, u^{*}, h^{\mathrm{L}}$ and $u^{\mathrm{L}}$ are mean values in sections $S^{*}$ (overflow) and $S^{\mathrm{L}}$ (lateral fluxes). Due to the symmetry of the problem, we only show one lateral flux. (b) Side view at a position $y$ of the flow overflowing the obstacle. We use a notation similar to that of the 2-D configuration given in Figure 1, but here the variables depend on the position, $y$.

2.4.2. Simplified analytical model for 3-D snow flows For the sake of simplicity and to provide an analytical model, we here consider the mean values over the obstacle width:

$$
\begin{aligned}
\bar{\alpha}_{\mathrm{sl}} & =\frac{1}{(\ell / 2)} \int_{0}^{\ell / 2} \alpha_{\mathrm{sl}}(y) \mathrm{d} y=\frac{\pi}{4}+\frac{\alpha_{\mathrm{sl}}}{2}, \\
\bar{\alpha}_{\mathrm{zm}} & =\frac{1}{(\ell / 2)} \int_{0}^{\ell / 2} \alpha_{\mathrm{zm}}(y) \mathrm{d} y, \\
\bar{\alpha} & =\frac{\bar{\alpha}_{\mathrm{sl}}+\bar{\alpha}_{\mathrm{zm}}}{2} .
\end{aligned}
$$

One parameter is introduced: $r=\ell_{a} / \ell$ is the ratio between the width of the incoming avalanche flow, $\ell_{a}$, and the width of the obstacle, $\ell$. Similarly to the 2-D case, we can apply the momentum balance over the control volume, $\bar{v}$. We simplify the calculation by taking the mean values over the obstacle width (given by Equations (11-13)) out of the 3-D integrals, which gives:

$$
\begin{gathered}
\left(\frac{F / \ell_{\mathrm{a}}}{\frac{1}{2} \rho u^{2} h}\right)_{u}=2 \beta\left[1-\frac{1}{r} \bar{\delta}_{\mathrm{u}}^{2} \bar{\delta}_{\mathrm{h}} \cos \bar{\alpha}\right. \\
\left.-\left(1-\frac{1}{r} \bar{\delta}_{\mathrm{u}} \bar{\delta}_{\mathrm{h}}\right) \bar{\delta}_{\mathrm{u}}^{\mathrm{L}} \cos \gamma\right], \\
\left(\frac{F / \ell_{\mathrm{a}}}{\frac{1}{2} \rho u^{2} h}\right)_{h+w-f}=\frac{1}{\mathrm{Fr}^{2}}\left\{k+\frac{1}{4 r}\left[\cos \theta+\left(\tan \theta_{\min }\right) \sin \theta\right]\right. \\
\left.\cdot\left(\frac{H}{h}+1+\frac{\bar{\delta}_{\mathrm{h}}}{\cos \bar{\alpha}}\right) \frac{H}{h}\right\} .
\end{gathered}
$$

We split the resulting ratio, $\left(F / \ell_{a}\right) /\left(0.5 \rho u^{2} h\right)$, into two parts: Equation (14) is the contribution due to the dynamic force and Equation (15) is the contribution of the sum of the incoming pressure force, the weight and the basal friction force. Three parameters have to be quantified: the mean ratio of velocities at the centre, $\bar{\delta}_{u}$, the mean ratio of the flow depths, $\bar{\delta}_{\mathrm{h}}$, and the mean ratio of velocities on both lateral sides, $\bar{\delta}_{u}^{\mathrm{L}}$. The mean lateral flow depths ratio, $\bar{\delta}_{\mathrm{h}}^{\mathrm{L}}=\bar{h}^{\mathrm{L}} / h$, where $h^{\mathrm{L}}$ is the thickness of lateral fluxes, is determined by the conservation of the mass flow rate: $1-\bar{\delta}_{u} \bar{\delta}_{\mathrm{h}}(1 / r)=$ $\bar{\delta}_{u}^{\mathrm{L}} \bar{\delta}_{\mathrm{h}}^{\mathrm{L}}[1-(1 / r)]$.

We use the following assumptions to derive simple empirical laws for these quantities. The mean ratio of velocities at the centre is calculated from the mean angle, $\bar{\alpha}: \bar{\delta}_{u}=1-\kappa \bar{\alpha}$, similarly to the 2-D geometry. The mean ratio of flow depths at the centre is assumed to be $\sim 1$, which corresponds to the assumption that the typical size of the overflowing flow is close to the typical size of the undisturbed flow. This is almost true for the case of 2-D granular flows (Faug and others, 2009) but it remains an assumption for 3-D flows, in the absence of well-documented experimental data. The mean ratio of velocities on both lateral sides is assumed to depend on the mean angle, $\gamma$ (by analogy with the definition of $\bar{\delta}_{\mathrm{u}}$ according to $\left.\bar{\alpha}\right): \bar{\delta}_{\mathrm{h}}^{\mathrm{L}}=1-\kappa^{\mathrm{L}} \gamma$, where we simply assume a value of $\kappa^{\mathrm{L}}$ equal to $\kappa=(1-e) /(\pi / 2)$. This latter assumption is argued by the fact that the restitution coefficients are similar for collisions in the planes $(x, z)$ and $(x, y)$. The angle $\gamma$ is defined as $\gamma=\arctan [(\ell / 2) / L]$ (Fig. 5a).

\section{MODEL PREDICTIONS COMPARED TO FULL-SCALE FIELD DATA}

Only few data corresponding to the geometry considered here are available in the literature. Three large-scale avalanche test sites provide some promising data. First, the Lautaret avalanche site in France, particularly the track equipped with a flat obstacle of surface equal to $1 \mathrm{~m}^{2}$ (Thibert and others, 2008), is of interest even if the geometry is complex due to lateral fluxes, jet and spreading effects. Second, the Vallée de la Sionne test site in Switzerland 

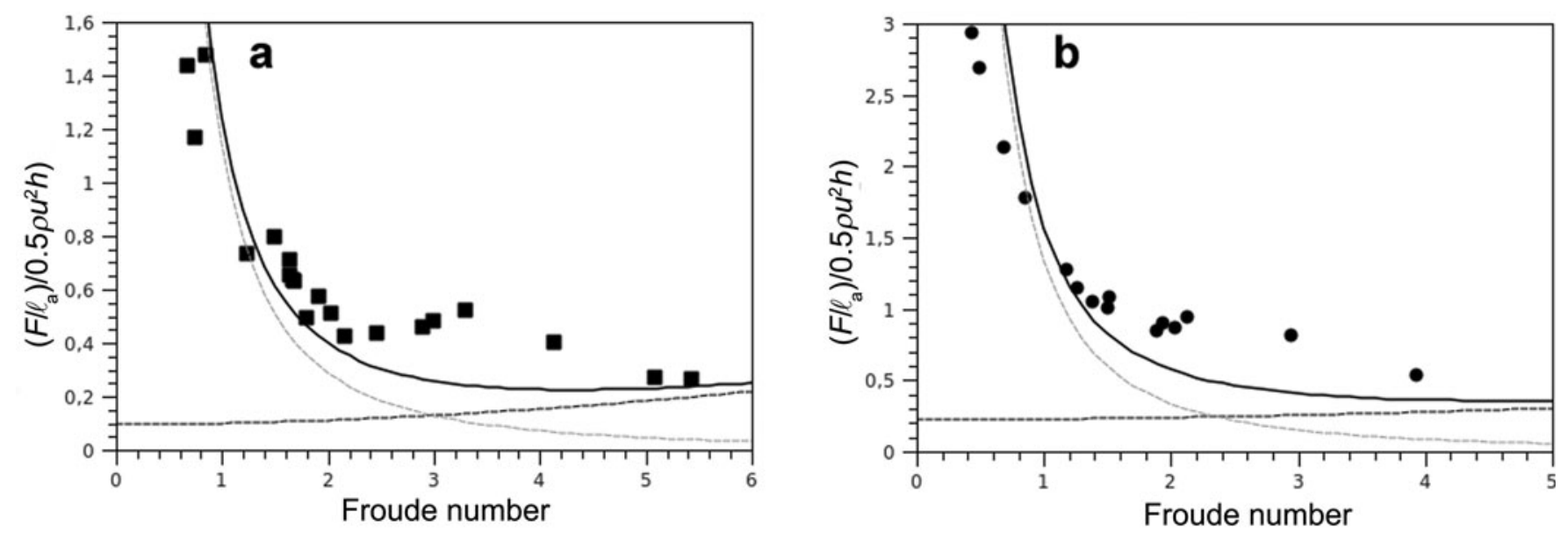

Fig. 5. Avalanche flows at Lautaret: predictions of the analytical model compared to the measured data (full black curve). The following parameters were used: $\beta=1, k=1, H / h=1, \theta_{\min }=33^{\circ}, \theta_{\max }=42^{\circ}, \xi=1000 \mathrm{~m} \mathrm{~s}^{-2}$ and $\kappa=\kappa^{\mathrm{L}}=(1-e) /(\pi / 2)$ with $e=0.1$. The grey dashed curve shows the contribution from the sum of the hydrostatic force, the gravity force and the basal friction force (Equation (15)). The black dashed curve shows the contribution from the incoming dynamic force (Equation (14)). (a) 15 February 2007 avalanche (Thibert and others, 2008) with $r=7$. (b) 26 March 2008 avalanche (Baroudi and Thibert, in press) with $r=3$.

provides well-documented data, but only obstacles that are small compared to the width of the avalanche flow (pylons or flat obstacles) are investigated (Sovilla and others, 2008b,a); these do not fit into the framework of our analytical model. Third, the Ryggfonn test site in Norway is of interest because it is close to the geometry of our analytical model, but it suffers from a lack of flow-depth data, implying an unknown Froude number (Faug and others, 2008b) and the dam is not normal to the incident flow.

The full-scale avalanche flows are transient flows that, a priori, are not compatible with our 3-D analytical model, for which we assume a steady state. As discussed above, we assume that the effects of time-derivative terms in the equations of motion are weak, so that a quasi-steady state is reached at each time, $t$, and that the basal friction, $\mu$, is close to $\tan \theta$. These assumptions allow us to express $\theta$ as a function of the incoming Froude number, where $\mu=\tan \theta_{\min }+(g / \xi) \mathrm{Fr}^{2}$ (Voellmy friction law). Then we can describe variations of $\left(F / \ell_{a}\right)\left(0.5 \rho u^{2} h\right)$ with $\mathrm{Fr}$.

Here we compare the predictions of the simplified 3-D model to the measurements from the Lautaret test site. One of the avalanche paths is equipped with a $1 \mathrm{~m}^{2}$ plate with pressure sensors. The avalanche site, the instruments and the procedure are presented in detail by Thibert and others (2008). In Figure 5, we compare the prediction from Equations (14) and (15) to the data for two avalanches from the Lautaret test site, 15 February 2007 (Thibert and others, 2008) and 26 March 2008 (Baroudi and Thibert, in press). Both avalanches were released in cold and dry snow conditions. Furthermore, the time-derivative terms were estimated and shown to be negligible for the 2007 avalanche in the decelerating-flow phase (see Thibert and others, 2008, fig. 11). We only consider data obtained in the decelerating-flow phase of the avalanche. The following set of parameters was used: $\beta=1, k=1, H / h=1, \theta_{\min }=3^{\circ}$, $\theta_{\max }=42^{\circ}, \xi=1000 \mathrm{~m} \mathrm{~s}^{-2}$ and $\kappa=\kappa^{\mathrm{L}}=(1-e) /(\pi / 2)$ with $e=0.1$. We used $r=7$ for the 15 February 2007 avalanche and $r=3$ for the 26 March 2008 avalanche. These values of $r$ are compatible with field observations from films taken of the avalanches. The 2007 avalanche was substantially larger than the 2008 avalanche. In spite of many assumptions, the prediction of our 3-D simplified model is in good agreement with the field data (full black curve in Fig. 5). We plot both contributions to the total force in Figure 5: one contribution from the dynamic force (Equation (14)) and the other contribution (Equation (15)) corresponding to the sum of the hydrostatic force, the gravity force and the basal friction. The graphs clearly show that the increase of the total rescaled force, $\left(F / \ell_{\mathrm{a}}\right) /\left(0.5 \rho u^{2} h\right)$, at low values of the Froude number $(\mathrm{Fr} \approx 1)$ is mainly due to the contribution corresponding to the sum of the hydrostatic force, the gravity force and the basal friction. Note that the increase of the rescaled force, $\left(F / \ell_{\mathrm{a}}\right) /\left(0.5 \rho u^{2} h\right)$, does not mean that the corresponding force, $F$, becomes the design force. For both avalanches investigated here, the force is maximal when the Froude number is higher (close to 4-5) and, in an engineering project, this maximal force would be the design force. By these graphs we demonstrate that when the avalanche comes to rest, the contributions from forces due to hydrostatic effect, weight and friction become dominant. This effect is particularly important in the run-out areas of snow avalanches. The simplified analytical model presented here has to be validated with more data. However, it can provide the ingredients for a practically applicable approximation of the total force exerted on obstacles by snow avalanches. When the design reference Froude number is low and large stagnant zones are likely to be formed upstream of the protection structure it is crucial to check whether the resulting force, $F$, calculated from the proposed model is greater than the force calculated using traditional engineering methods (Salm and others, 1990).

\section{DISCUSSION AND CONCLUSION}

We have presented an analytical model to estimate the force on flat obstacles when a stagnant zone is formed upstream of the obstacle. We first presented a 2-D analytical model describing gravity-driven flows overflowing a wall normal to the ground. Three important parameters were needed to close the model: the friction, $\mu_{\mathrm{zm}}$, between the dead zone and the ground, the coefficient of velocity reduction, $\kappa$, and the angle of the free-surface upstream of the wall, $\alpha$. The 
analytical 2-D model and the empirical laws proposed to quantify these parameters were validated by discrete-particle simulations of granular flows down an inclined slope. We provided the parameters to use this analytical model for 2-D flows of dry snow, which allowed us to quantify the effect of the stagnant zone on the resulting force, particularly at low incoming Froude numbers, and to provide a tool to estimate the force on large catching dams with no (or little) lateral overflows. This 2-D analytical model was then extended to a 3 -D configuration in order to predict the force when lateral fluxes occur. The analytical model was given for steady-flow conditions but we believe that it is also suitable for transient flows for which the effect of time-derivative terms can be neglected. This is typically the case for snow avalanches in the decelerating phase (before the final standstill). We compared the prediction of our analytical model to the field data available at Lautaret. We only used the data in the quasi-steady state corresponding to the decelerating phase (Thibert and others, 2008, fig. 11). The analytical predictions are in good agreement with field observations within the experimental uncertainty. More validation of the analytical model is needed (not only for the force, but also for geometrical data, such as the deflecting angles and the shape of the stagnant zone). Let us stress the fact that the time dependency of avalanche flows has not been considered in this paper, which means that our proposed model is not able to predict acting transient forces on obstacles induced by the avalanche front. Further work is needed to analyse these transient forces and the related fluctuations. Furthermore, a sensitivity analysis of the model with respect to all the parameters needs to be performed before we can propose that the sketched methodology serve as conceptional base for a practioners' recipe to estimate design forces for structures that can be hit by avalanches. The parameter combinations for various avalanche types, similar to those of Salm and others (1990) for the estimation of avalanche run-out lengths and velocities with the Voellmy friction law, could be used to carry out this task. However, we recommend investigating the force at low Froude numbers, when stagnant-zone mechanisms are likely to occur, at a more fundamental level.

\section{ACKNOWLEDGEMENTS}

We thank H. Bellot, F. Ousset and X. Ravanat for their work at the Lautaret test site. Thanks also to D. Bertrand who helped in performing the numerical simulations with the commercial code PFC2D (Itasca Consulting: http://www.itasca.com/pfc/index.php).

\section{REFERENCES}

Baroudi, D. and E. Thibert. In press. An instrumented structure to measure avalanche impact pressure: error analysis from Monte Carlo simulations. Cold Reg. Sci. Technol. (10.1016/j.coldregions.2009.05.010.)

Bartelt, P., B. Salm and U. Gruber. 1999. Calculating densesnow avalanche runout using a Voellmy-fluid model with active/passive longitudinal straining. J. Glaciol., 45(150), 242254.

Bouchet, A., M. Naaim, F. Ousset, H. Bellot and D. Cauvard. 2003. Experimental determination of constitutive equations for dense and dry avalanches: presentation of the set-up and first results. Surv. Geophys. , 24(5-6), 525-541.
Bouchet, A., M. Naaim, H. Bellot and F. Ousset. 2004. Experimental study of dense snow avalanches: velocity profiles in steady and fully developed flows. Ann. Glaciol., 38, 30-34.

Chiou, M.-C., Y. Wang and K. Hutter. 2005. Influence of obstacles on rapid granular flows. Acta Mech., 175(1-4), 105-122.

Chu, T., G. Hill, D.M. McClung, R. Ngun and R. Sherkat. 1995. Experiments on granular flows to predict avalanche runup. Can. Geotech. J., 32(2), 285-295.

Cui, X., J.M.N.T. Gray and T. Jóhannesson. 2007. Deflecting dams and the formation of oblique shocks in snow avalanches at Flateyri, Iceland. J. Geophys. Res., 112(F4), F04012. (10.1029/2006JF000712.)

Cundall, P.A. and O.D.L. Strack. 1979. A discrete numerical model for granular assemblies. Géotechnique, 29(1), 47-65.

Da Cruz, F., S. Emam, M. Prochnow, J.-N. Roux and F. Chevoir. 2005. Rheophysics of dense granular materials: discrete simulation of plane shear flows. Phys. Rev. E, 72, 021309. (10.1103/PhysRevE.72.021309.)

Ertas, D., G.S. Grest, T.C. Halsey, D. Levine and L.E. Silbert. 2001. Gravity-driven dense granular flows. Europhys. Lett., 56(2), 214-220.

Faug, T., P. Lachamp and M. Naaim. 2002. Experimental investigation on steady granular flows interacting with an obstacle down an inclined channel: study of the dead zone upstream from the obstacle. Application to interaction between dense snow avalanches and defence structures. Natur. Hazards Earth Syst. SCi. (NHESS), 2(3/4), 187-191.

Faug, T., M. Naaim and A. Fourrière. 2007. Dense snow flowing past a deflecting obstacle: an experimental investigation. Cold Reg. Sci. Technol., 49(1), 64-73.

Faug, T., B. Chanut, M. Naaim and B. Perrin. 2008a. Avalanches overflowing a dam: dead zone, granular bore and run-out shortening. Ann. Glaciol., 49, 77-82.

Faug, T., P. Gauer, K. Lied and M. Naaim. 2008b. Overrun length of avalanches overtopping catching dams: cross-comparison of small-scale laboratory experiments and observations from full-scale avalanches. J. Geophys. Res., 113(F3), F03009. (10.1029/2007JF000854.)

Faug, T., R. Beguin and B. Chanut. 2009. Mean steady granular force on a wall overflowed by free-surface gravity-driven flows in steady and unsteady states. Phys. Rev. E, 80, 021305. (10.1103/PhysRevE.80.021305.).

Gauer, P. and 7 others. 2007. On full-scale avalanche measurements at the Ryggfonn test site, Norway. Cold Reg. Sci. Technol., 49(1), 39-53.

Gauer, P., K. Lied and K. Kristensen. 2009. Analysis of avalanche measurements out of the runout area of NGI's full-scale test-site Ryggfonn. Cold Reg. Sci. Technol., 57(1), 1-6.

GDR MiDi, 2004. On dense granular flows. Eur. Phys. J. E, 14(4), 341-365.

Gray, J.M.N.T. and X. Cui. 2007. Weak, strong and detached oblique shocks in gravity-driven granular free-surface flows. J. Fluid Mech., 579, 113-136.

Gray, J.M.N.T., Y.-C. Tai and S. Noelle. 2003. Shock waves, dead zones and particle-free regions in rapid granular free-surface flows. J. Fluid Mech., 491, 161-181.

Hákonardóttir, K.M. and A.J. Hogg. 2005. Oblique shocks in rapid granular flows. Phys. Fluids, 17, 077101. (10.1063/1.1950688.)

Hákonardóttir, K.M., A.J. Hogg, T. Jóhannesson and G.G. Tómasson. 2003. A laboratory study of the retarding effects of braking mounds on snow avalanches. J. Glaciol., 49(165), 191-200.

Irgens, F., B. Schieldrop, C.B. Harbitz, U. Domaas and R. Opsahl. 1998. Simulations of dense-snow avalanches on deflecting dams. Ann. Glaciol., 26, 265-271.

Jóhannesson, T. 2001. Run-up of two avalanches on the deflecting dams at Flateyri, northwestern Iceland. Ann. Glaciol., 32, 350-354.

McClung, D.M. and A.I. Mears. 1995. Dry-flowing avalanche runup and run-out. J. Glaciol., 41(138), 359-372. 
Naaim, M., F. Naaim-Bouvet, T. Faug and A. Bouchet. 2004. Dense snow avalanche modeling: flow, erosion, deposition and obstacle effects. Cold Reg. Sci. Technol., 39(2-3), 193-204.

Pouliquen, O. 1999. Scaling laws in granular flows down rough inclined planes. Phys. Fluids, 11(3), 542-548.

Rognon, P. 2006. Rhéologie des milieux granulaires cohésifs application aux avalanches de neige dense. (PhD thesis, Ecole Nationale des Ponts et Chaussées.)

Rognon, P.G., F. Chevoir, H. Bellot, F. Ousset, M. Naaim and P. Coussot. 2008. Rheology of dense snow flows: inferences from steady state chute-flow experiments. J. Rheol., 52(3), 729-748.

Salm, B., A. Burkard and H. Gubler. 1990. Berechnung von Fliesslawinen: eine Anleitung für Praktiker mit Beispielen. Eidg. Inst. Schnee- und Lawinenforsch. Mitt. 47.

Savage, S.B. and K. Hutter. 1989. The motion of a finite mass of granular material down a rough incline. J. Fluid Mech., 199, 177-215.
Silbert, L.E., D. Ertas, G.S. Grest, T.C. Halsey, D. Levine and S.J. Plimpton. 2001. Granular flow down an inclined plane: Bagnold scaling and rheology. Phys. Rev. E, 64(5), 051302. (10.1103/PhysRevE.64.051302.)

Sovilla, B., M. Schaer, M. Kern and P. Bartelt. 2008a. Impact pressures and flow regimes in dense snow avalanches observed at the Vallée de la Sionne test site. J. Geophys. Res., 113(F1), F01010. (10.1029/2006JF000688.)

Sovilla, B., M. Schaer and L. Rammer. 2008b. Measurements and analysis of full-scale avalanche impact pressure at the Vallée de la Sionne test site. Cold Reg. Sci. Technol., 51(2-3), 122-137.

Tai, Y.C., J.M.N.T. Gray, K. Hutter and S. Noelle. 2001. Flow of dense avalanches past obstructions. Ann. Glaciol., 32, 281-284.

Thibert, E., D. Baroudi, A. Limam and P. Berthet-Rambaud. 2008. Avalanche impact pressure on an instrumented structure. Cold Reg. Sci. Technol., 54(3), 206-215. 